\title{
Short communication \\ Oxygen mass transfer in a high solids loading three-phase internal-loop airlift reactor
}

\author{
Carla Freitas, José A. Teixeira* \\ Centro de Engenharia Biológica-IBQF, Universidade do Minho, Campus de Gualtar, 4710-057 Braga, Portugal
}

Received 18 April 2000; received in revised form 2 October 2000; accepted 4 October 2000

\begin{abstract}
Determinations of volumetric mass transfer coefficient were conducted in a three-phase internal-loop airlift reactor with an enlarged degassing zone. The effect of parameters such as the airflow rate (riser superficial gas velocities between 0.01 and $0.5 \mathrm{~m} / \mathrm{s}$ ), solids loading (up to $30 \%, v / v)$, solids density (1023 and $1048 \mathrm{~kg} / \mathrm{m}^{3}$ ) and the liquid-phase properties on $k_{\mathrm{L}} a$ was studied. It was observed that the increase of the airflow rate and the introduction of ethanol enhanced the volumetric mass transfer coefficient in the system. On the contrary, the progressive introduction of solids and a small increase on solids density were responsible for the diminishing of the mass transfer rate. Correlations for the volumetric mass transfer coefficient with the riser superficial gas velocity and solids loading were determined for the two solids density and the two liquid-phases. A good agreement between experimental data and the calculated values was obtained. (C) 2001 Elsevier Science B.V. All rights reserved.
\end{abstract}

Keywords: Airlift reactor; Mass transfer; Solids density; Solids loading; Liquid-phase properties

\section{Introduction}

The primary purpose of a bioreactor is to provide such environmental conditions to the microorganism that it will carry out the required reaction or transformation optimally [1]. In aerobic bioreactors, the critical limiting factor in providing the optimal environment is the oxygen mass transfer, as a consequence of the low solubility of oxygen in fermentation media. Hence, the volumetric mass transfer coefficient $\left(k_{\mathrm{L}} a\right)$, which is normally used to characterize the mass transfer performance, plays an important role in the performance of bioreactors [1-3]. Ideally, a reactor should have a maximum mass transfer rate, at an efficient mixing and at a minimum energy input [2]. Several studies have demonstrated the airlift reactors ability to provide this $[1,2,4]$. However, the data presented in the literature for mass transfer in airlift reactors vary widely and is sometimes contradictory due, probably, to the wide range of reactors studied and differences in experimental techniques used [2,3]. Most experimental measurements [5,6] have shown that the rate of mass transfer in an airlift reactor is

\footnotetext{
* Corresponding author. Tel.: +351-253-604-400; fax: +351-253-678-986.

E-mail address: jateixeira@deb.uminho.pt (J.A. Teixeira).
}

influenced by various design (i.e., bioreactor type and geometry), system (i.e., fluid properties) and operation (i.e., liquid and gas velocity and gas holdup) variables.

Fluid coalescence properties can vary between two extreme cases: coalescing and non-coalescing. Fermentation liquids will always be between the two extremes. However, due to their complex composition, it is impossible to predict where "between" [5]. Once released from the sparger, the bubbles are subjected to the dispersion and the coalescence processes, what will influence their size. Therefore, studies have shown that liquids differing from water on the surface tension, density, viscosity and ionic strength affect gas holdup and the overall mass transfer coefficient [1], being its influence dependent on the reactor type.

The use of gas-liquid-solid three-phase reactors has been widespread in chemical processing and those containing low-density particles are finding applications in biotechnology processes such as fermentation and aerobic wastewater treatment [6]. The solids suspended in biological processes, including microorganisms immobilized on solid-support particles and aggregated in flocs and pellets, have, in general, a density very close to that of the liquid medium, which may, sometimes, change during the process. However, few studies have addressed three-phase airlift reactors with low density solids [6-8] and none known so far reported the 


\begin{tabular}{|ll|}
\hline \multicolumn{2}{|l|}{ Nomenclature } \\
$a$ & gas-liquid interfacial area per unit \\
& liquid volume $(1 / \mathrm{m})$ \\
$A_{\mathrm{d}}$ & downcomer cross-sectional area $\left(\mathrm{m}^{2}\right)$ \\
$A_{\mathrm{r}}$ & riser cross-sectional area $\left(\mathrm{m}^{2}\right)$ \\
$C_{\mathrm{L}}$ & instantaneous concentration of oxygen \\
& in liquid $\left(\mathrm{kg} / \mathrm{m}^{3}\right)$ \\
$C_{\mathrm{L} 0}$ & initial concentration of oxygen in \\
& the liquid $\left(\mathrm{kg} / \mathrm{m}^{3}\right)$ \\
$C^{*}$ & saturation $($ or equilibrium) dissolved \\
& oxygen concentration $\left(\mathrm{kg} / \mathrm{m}^{3}\right)$ \\
$k_{\mathrm{L}}$ & mass transfer coefficient $(\mathrm{m} / \mathrm{s})$ \\
$t$ & time (s) \\
$t_{\mathrm{E}}$ & electrode delay $(\mathrm{s})$ \\
$u_{\mathrm{gr}}$ & riser superficial gas velocity $(\mathrm{m} / \mathrm{s})$ \\
& \\
Greek & symbol \\
$\varepsilon_{\mathrm{S}}$ & solids loading \\
\hline
\end{tabular}

influence of small increases on the solids density in the mass transfer rate.

The objective of the present work was to examine the way volumetric mass transfer coefficient in an internal-loop airlift reactor with a degassing zone is influenced by changing system and operation variables: the liquid-phase, the solids loading, the solids density and the airflow rate. These were shown in previous works $[9,10]$ to influence other hydrodynamic variables directly related with the mass transfer as the liquid velocity, mixing and gas holdup.

\section{Experimental}

Experiments were performed in a 601 internal-loop airlift reactor of the concentric tube type, with an enlarged degassing zone, described by Freitas and Teixeira [9]. This reactor was constructed according to previous results obtained by Vicente and Teixeira [11] in what concerns the effect of design parameters on solid circulation. The airflow rate was varied in a way that the riser superficial gas velocities studied were between 0.01 and $0.5 \mathrm{~m} / \mathrm{s}$.

Water and a $10 \mathrm{~g} / \mathrm{l}$ aqueous solution of ethanol were used as liquid-phase and Ca-alginate beads, with two different densities, were used as solid-phase and prepared according to the procedure described by Vicente and Teixeira [11]. The mean diameter and density of the low density solids (LD) were $2.131 \pm 0.102 \mathrm{~mm}$ and $1023 \pm 1 \mathrm{~kg} / \mathrm{m}^{3}$, respectively, and the values for the high density solids (HD) were $2.151 \pm$ $0.125 \mathrm{~mm}$ and $1048 \pm 1 \mathrm{~kg} / \mathrm{m}^{3}$. For each type of solids, solids loading applied was $0,5,10,15,20$ and $30 \%(\mathrm{v} / \mathrm{v})$.

The concentration of the dissolved oxygen in the liquid was measured continuously by means of two polarographic dissolved oxygen electrodes (Mettler Toledo T-type) connected to dissolved oxygen meters (Mettler Toledo $\mathrm{O}_{2}$ 4100) and recorded using a $\mathrm{PC}$ based data acquisition system. The volumetric mass transfer coefficient $k_{\mathrm{L}} a$ was determined by the dynamic method described by Chisti [1] and Benyhaia et al. [3]. In this work, values of the volumetric mass transfer coefficients $\left(k_{\mathrm{L}} a\right)$ were obtained from the first order model [1]:

$$
\frac{C^{*}-C_{\mathrm{L}}}{C^{*}-C_{\mathrm{L} 0}}=1-E=\left(\frac{\mathrm{e}^{-t k_{\mathrm{L}} a}}{t_{\mathrm{E}}}-k_{\mathrm{L}} a \mathrm{e}^{-\left(t / t_{E}\right)}\right) \frac{t_{\mathrm{E}}}{1-t_{\mathrm{E}} k_{\mathrm{L}} a}
$$

where $E$ is defined as

$E=\frac{C_{\mathrm{L}}-C_{\mathrm{L} 0}}{C^{*}-C_{\mathrm{L} 0}}$

and $t_{\mathrm{E}}$ is the electrode time delay. For $t \gg t_{\mathrm{E}}$, Eq. (1) reduces to

$$
1-E=\frac{\mathrm{e}^{-t k_{\mathrm{L}} a}}{1-t_{\mathrm{E}} k_{\mathrm{L}} a}
$$

According to Van't Riet [12], the electrode response time would have to be much smaller than the mass transfer response time of the system, $1 / k_{\mathrm{L}} a$, to be neglected. In practice, this is seldom the case.

For each experimental condition, three replicates were done and the volumetric mass transfer coefficient was the medium value of the values obtained for the two electrodes in the three replicates.

\section{Results and discussion}

In this work, the presence of the solid phase negatively influences the mass transfer rate (Fig. 1a and b), as was reported by other authors $[6,13,14]$. Independently of the liquid-phase and the solids density, $k_{\mathrm{L}} a$ diminishes with the increase of solids loading, essentially for riser superficial gas velocities higher than $0.075 \mathrm{~m} / \mathrm{s}$. The reduction on the volumetric mass transfer coefficient is probably the result of the coalescence process, increased by the increase of the amount of solids as reported in Freitas and Teixeira [9], what leads to a decrease on the interfacial area $a$. According to Verlaan and Tramper [7], the effect on the mass transfer coefficient $k_{\mathrm{L}}$ will be of minor importance.

Up to $0.075 \mathrm{~m} / \mathrm{s}$, the effect of solids loading on $k_{\mathrm{L}} a$ is negligible, once the amount of gas in the reactor is so small that the solids interference in the coalescence process is not significant.

Comparing to the works of Siegel et al. [17] and Komáromy and Sisak [14], where reductions of $25 \%$ were obtained with solids loading of 20 and 30\%, respectively, the reductions of $k_{\mathrm{L}} a$ in the present work are very high. Reductions between 40 and $60 \%$ were obtained for the higher solids loading.

From Fig. 1, it can be concluded about the effect of the addition of a coalescence inhibitor on the volumetric mass 


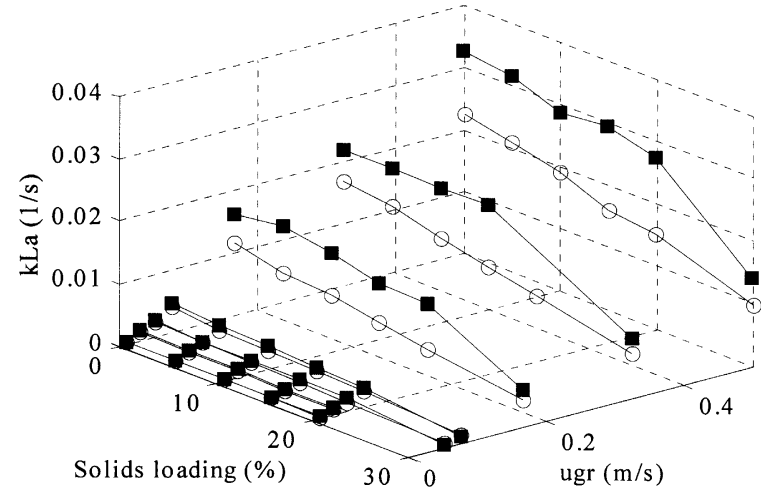

(a)

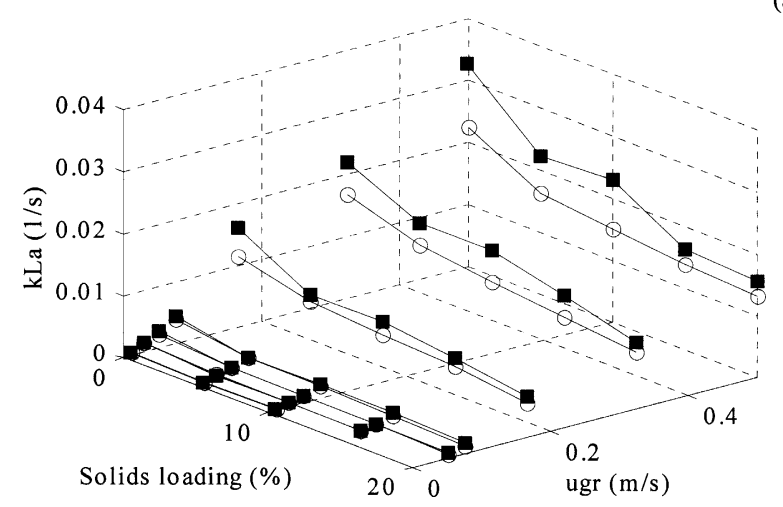

(b)

Fig. 1. Volumetric mass transfer coefficient $\left(k_{\mathrm{L}} a\right)$ vs. solids loading and riser superficial gas velocity $\left(u_{\mathrm{gr}}\right)$, for water $(\mathrm{O})$ and the aqueous ethanol solution (ם): (a) low density solids; (b) high density solids.

transfer coefficient. It is shown that by adding ethanol to water the volumetric mass transfer coefficient is significantly enhanced for riser superficial gas velocities higher than $0.075 \mathrm{~m} / \mathrm{s}$. This increase on $k_{\mathrm{L}} a$ must be due to an increase in specific interfacial area, since with the addition of alcohols the mass transfer coefficient $k_{\mathrm{L}}$ decreases, by yielding an additional resistance to the interfacial mass transfer [17]. As alcohols are inhibitors of coalescence, bubble size decreases and the specific interfacial area increases.

According to Onken and Weiland [18] and Weiland [20], the addition of compounds that reduce bubble coalescence increases the volumetric mass transfer coefficient by a factor of about 2. In our case, the increase of $k_{\mathrm{L}} a$ depends on the amount and type of solids and on the airflow rate. For riser superficial gas velocities up to $0.075 \mathrm{~m} / \mathrm{s}$, the increase is very small, while for higher airflow rates, values of $k_{\mathrm{L}} a$ for aqueous ethanol solution are 1.5-2 times the values obtained with water.

The effect of the airflow rate and solids density on the volumetric mass transfer coefficient $\left(k_{\mathrm{L}} a\right)$ can be seen in Fig. 2, for water (a) and aqueous ethanol solution (b). The $k_{\mathrm{L}} a$ value increases with an increasing riser superficial gas velocity $\left(u_{\mathrm{gr}}\right)$, which is due to the higher gas holdup, as was reported in Freitas and Teixeira $[9,10]$. As for the gas holdup, the increase of $k_{\mathrm{L}} a$ is more pronounced for values
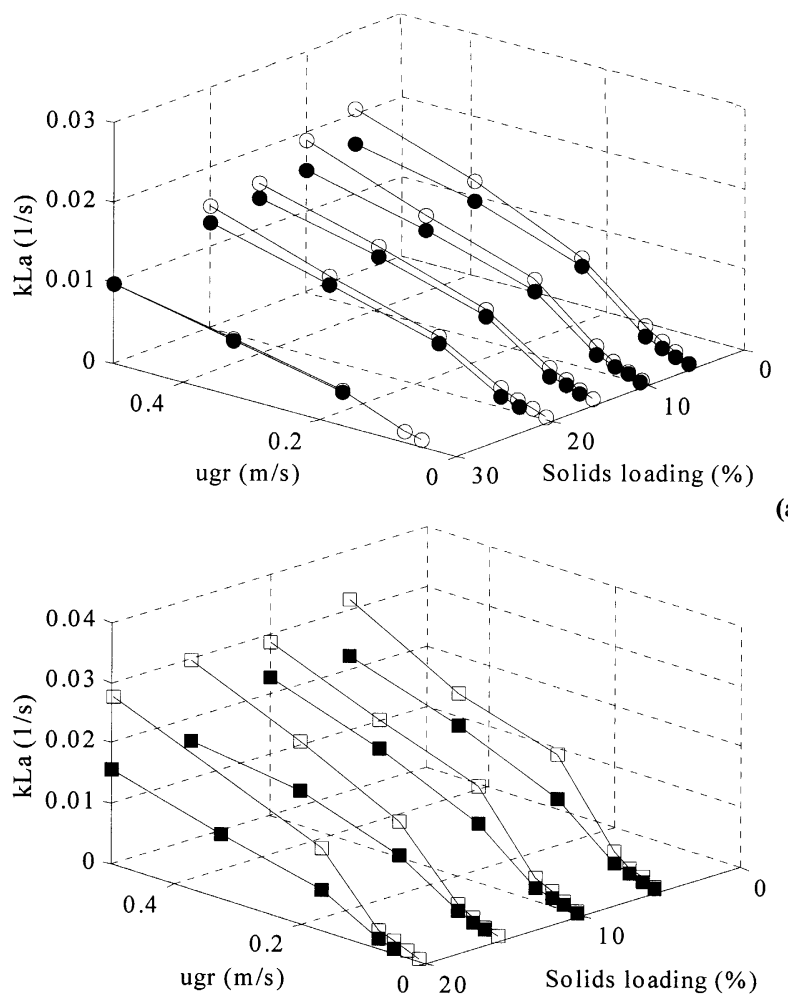

(a)

Fig. 2. Volumetric mass transfer coefficient $\left(k_{\mathrm{L}} a\right)$ vs. solids loading and riser superficial gas velocity $\left(u_{\mathrm{gr}}\right)$, for the two types of solids $((\bigcirc, \square)$ low density; (, $\mathbf{\square})$ high density): (a) water; (b) aqueous ethanol solution.

of superficial gas velocity up to $0.167 \mathrm{~m} / \mathrm{s}$. In spite of the higher amount of gas inside the reactor as the airflow rate increases, coalescence occurs more easily for high airflow rates, counterbalancing the increase on gas holdup.

Observing values of mass transfer coefficient for both solids density, it can be seen that a small increase on solids density leads to a significant reduction on $k_{\mathrm{L}} a$ (Fig. 2), specially for high riser superficial gas velocities. An enhancement of riser and downcomer solids holdup with increasing solids density was observed in a previous work [9]. For that reason, coalescence increases due to the diminishing of the flux area for the gas and liquid-phases, which is reflected in a smaller interfacial area $a$. If we compare the influence of solids density on the volumetric mass transfer coefficient for water (Fig. 2a) and aqueous ethanol solution (Fig. 2b), it seems that the decrease of $k_{\mathrm{L}} a$ with solids density is higher for the aqueous ethanol solution. This is in agreement with the results previously obtained by the authors [10], where it was observed that the decrease of riser and downcomer gas holdup with solids density is also more pronounced for the ethanol solution, being this reflected in the results of volumetric mass transfer coefficient.

The volumetric mass transfer coefficient was correlated to the riser superficial gas velocity $\left(u_{\mathrm{gr}}\right)$ and the solids loading $\left(\varepsilon_{\mathrm{s}}\right)$. Four generalized equations were found including 

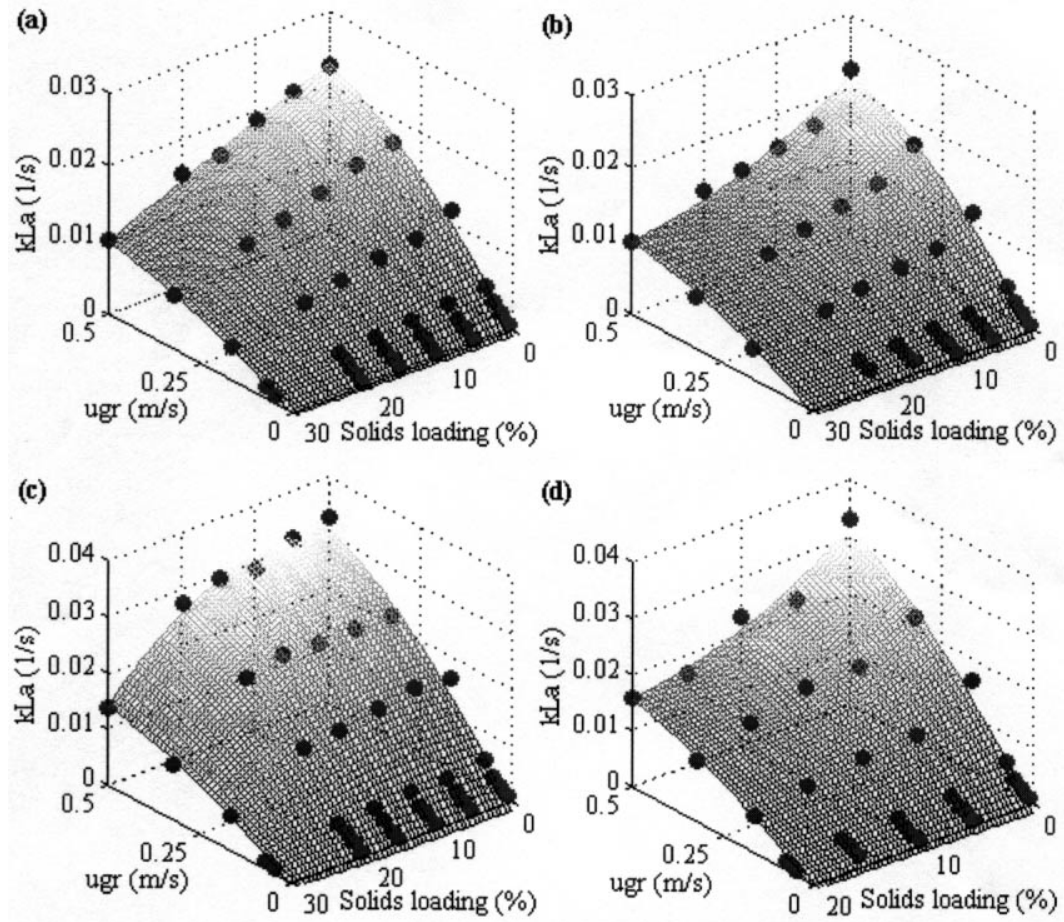

Fig. 3. Representation of the relation between the volumetric mass transfer coefficient $\left(k_{\mathrm{L}} a\right)$ with the riser superficial gas velocity $\left(u_{\mathrm{gr}}\right)$ and the solids loading ((O) experimental data; surface representation — values calculated by the correlations): (a) water/low density solids; (b) water/high density solids; (c) aqueous ethanol solution/low density solids; (d) aqueous ethanol solution/high density solids.

these two process variables, by fitting all of the data, for each liquid-phase and type of solids. In literature [15-17,19] relations of the type $k_{\mathrm{L}} a=a u_{\mathrm{gr}}^{b}$ and of the type $k_{\mathrm{L}} a=$ $a \varepsilon_{\mathrm{s}}^{2}+b \varepsilon_{\mathrm{S}}+c$, between the volumetric mass transfer coefficient and the riser superficial gas velocity and the solids loading, respectively, are generally used. However, a quadratic equation for both $u_{\mathrm{gr}}$ and $\varepsilon_{\mathrm{s}}$ was found necessary to provide an acceptable fit. The obtained correlations were:

Water/low density solids:

$$
\begin{aligned}
k_{\mathrm{L}} a= & \left(-0.93 u_{\mathrm{gr}}^{2}+1.33 u_{\mathrm{gr}}-0.012\right) \\
& \times\left(-0.0000016 \varepsilon_{\mathrm{s}}^{2}-0.00099 \varepsilon_{\mathrm{s}}+0.054\right)
\end{aligned}
$$

Water/high density solids:

$$
\begin{aligned}
k_{\mathrm{L}} a= & \left(-0.33 u_{\mathrm{gr}}^{2}+0.43 u_{\mathrm{gr}}-0.0064\right) \\
& \times\left(0.000080 \varepsilon_{\mathrm{s}}^{2}-0.0056 \varepsilon_{\mathrm{s}}+0.17\right)
\end{aligned}
$$

Ethanol/low density solids:

$$
\begin{aligned}
k_{\mathrm{L}} a= & \left(-0.95 u_{\mathrm{gr}}^{2}+1.34 u_{\mathrm{gr}}-0.021\right) \\
& \times\left(-0.000072 \varepsilon_{\mathrm{s}}^{2}+0.00079 \varepsilon_{\mathrm{s}}+0.075\right)
\end{aligned}
$$

Ethanol/high density solids:

$$
\begin{aligned}
k_{\mathrm{L}} a= & \left(-0.78 u_{\mathrm{gr}}^{2}+1.20 u_{\mathrm{gr}}-0.021\right) \\
& \times\left(-0.000074 \varepsilon_{\mathrm{s}}^{2}-0.0035 \varepsilon_{\mathrm{s}}+0.081\right)
\end{aligned}
$$

where

$$
0<u_{\text {gr }}(\mathrm{m} / \mathrm{s})<0.5, \quad 0<\varepsilon_{\mathrm{s}}(\%, \mathrm{v} / \mathrm{v})<30
$$

The points given by the correlations together with the experimental values are represented in Fig. 3. As can be seen, a good agreement $( \pm 10 \%$ for water and $\pm 20 \%$ for aqueous ethanol) is obtained between calculated and experimental values. The low $k_{\mathrm{L}} a$ values reported in this work compared to those in the literature can be justified by the use of a downcomer to riser cross-sectional area ratio $\left(A_{\mathrm{d}} / A_{\mathrm{r}}\right)$ greater than 1 [17].

\section{Conclusions}

The volumetric mass transfer coefficient was determined for a three-phase internal-loop airlift reactor with an enlarged degassing zone.

It was shown that the volumetric mass transfer coefficient diminishes with the increase of solids loading, especially for high airflow rates, due to an increase in bubble coalescence. Reductions between 40 and $70 \%$ were obtained with the introduction of 20 and $30 \%$ (v/v) of solids. Solids density also affects $k_{\mathrm{L}} a$. A significant decrease on $k_{\mathrm{L}} a$ resulting from a small increase of solids density (from 1023 to $1048 \mathrm{~kg} / \mathrm{m}^{3}$ ) was observed, as a consequence of the effect of solids density on solids distribution in the reactor. A progressive increase on the concentration of solids in the lower sections of the 
reactor, as riser and downcomer, with the increase of solids density is responsible for the enhancement of coalescence and the consequent decrease on the specific interfacial area.

Airflow rate and the presence of ethanol were shown to enhance the volumetric mass transfer coefficient - airflow rate by primarily increasing gas holdup and ethanol by inhibiting coalescence. The increase on $k_{\mathrm{L}} a$ by the presence of ethanol depends on the amount and type of solids and on the airflow rate.

The volumetric mass transfer coefficient was correlated to the riser superficial gas velocity $\left(u_{\mathrm{gr}}\right)$ and the solids loading $\left(\varepsilon_{\mathrm{s}}\right)$, for the two types of solids and for the two liquids, water and ethanol solution. Agreements between the calculated values and the experimental data of $\pm 10 \%$, for water, and of $\pm 20 \%$, for aqueous ethanol solution, were obtained.

\section{Acknowledgements}

This work was supported by FCT (Fundação para a Ciência e a Tecnologia), under project PRAXIS/2/2.1/BIO/ $1061 / 95$ and grant GGP XXI/BD/2937/96 awarded to Carla Maria Duarte de Freitas and by EC, under INCO-COPERNICUS contract No. ERB IC15-CT98-0904. The authors also acknowledge the financial support provided by the Instituto de Biotecnologia e Química Fina (IBQF).

\section{References}

[1] M.Y. Chisti, Airlift Bioreactors, Elsevier Applied Science, London, UK, 1989.

[2] J.C. Merchuk, M.H. Siegel, Airlift reactors in chemical and biological technology, J. Chem. Technol. Biotechnol. 41 (1988) 105-120.

[3] F. Benyhaia, L. Jones, D. Plantaz, Mass transfer studies in pneumatic reactors, Chem. Eng. Technol. 19 (1996) 425-431.

[4] M.H. Siegel, J.C. Merchuk, Mass transfer in a rectangular airlift reactor: effects of geometry and gas recirculation, Biotechnol. Bioeng. 32 (1988) 1128-1137.

[5] K. Van't Riet, J. Tramper, Basic Bioreactor Design, Marcel Dekker, New York, 1991.
[6] Y. Kawase, N. Hashimoto, Gas holdup and oxygen transfer in three-phase external-loop airlift bioreactors: non-Newtonian fermentation broths, J. Chem. Technol. Biotechnol. 65 (1996) 325-334.

[7] P. Verlaan, J. Tramper, Hydrodynamics, axial dispersion and gas-liquid oxygen transfer in an airlift-loop bioreactor with three-phase flow, in: Proceedings of the International Conference on Bioreactors and Biotransformations, Scotland, November 1987, pp. 363-373.

[8] H.-L. Tung, Y.-Y. Chang, T.-K. Hwang, W.-T. Wu, Liquid mixing and mass transfer in a modified bubble column with suspended particles, J. Chin. Inst. Chem. Engrs. 29 (1998) 467-472.

[9] C. Freitas, J.A. Teixeira, Hydrodynamic studies in an airlift reactor with an enlarged degassing zone, Bioprocess. Eng. 18 (1997) 267-279.

[10] C. Freitas, J.A. Teixeira, Effect of liquid-phase surface tension on hydrodynamics of a three-phase airlift reactor with an enlarged degassing zone, Bioprocess. Eng. 19 (1998) 451-457.

[11] A.A. Vicente, J.A. Teixeira, Hydrodynamic performance of a threephase airlift bioreactor with an enlarged degassing zone, Bioprocess. Eng. 14 (1995) 17-22.

[12] K. Van't Riet, Review of measuring methods and results in non-viscous gas-liquid mass transfer in stirred vessels, Ind. Eng. Chem. Process. Des. Dev. 18 (1979) 357-364.

[13] C. Nicolella, M. van Loosdrecht, R. van der Lans, J. Heijnen, Hydrodynamic characteristics and gas-liquid mass transfer in a biofilm airlift suspension reactor, Biotechnol. Bioeng. 60 (1998) 627-635.

[14] P. Komáromy, C. Sisak, Investigation of gas-liquid oxygen transport in three-phase bioreactor, Hung. J. Ind. Chem. 22 (1994) 147-151.

[15] W. Bang, I. Nikov, H. Delmas, A. Bascoul, Gas-liquid mass transfer in a new three-phase stirred airlift reactor, J. Chem. Technol. Biotechnol. 72 (1998) 137-142.

[16] B. Smith, D. Skidmore, Mass transfer phenomena in an airlift reactor: effects of solids loading and temperature, Biotechnol. Bioeng. 35 (1990) 483-491.

[17] M.H. Siegel, M. Hallaile, M. Herskowitz, J.C. Merchuk, Hydrodynamics and mass transfer in a three-phase airlift reactor, in: R. King (Ed.), Proceedings of the Second International Conference on Bioreactor Fluid Dynamics, September 1988, pp. 337-352.

[18] U. Onken, P. Weiland, Hydrodynamics and mass transfer in an airlift loop fermentor, Eur. J. Appl. Microbiol. Biotechnol. 10 (1980) 31-40.

[19] K. Kawase, N. Hashiguchi, Gas-liquid mass transfer in external-loop airlift columns with Newtonian and non-Newtonian fluids, Chem. Eng. J. 62 (1996) 35-42.

[20] P. Weiland, Influence of draft tube diameter on operation behaviour of airlift loop reactors, Ger. Chem. Eng. 7 (1984) 374-385. 